Voix et Images

volxetimages

\title{
Michel Tremblay : Du texte à la représentation. Entretien
}

\section{Roch Turbide}

Volume 7, numéro 2, hiver 1982

Michel Tremblay

URI : https://id.erudit.org/iderudit/200319ar

DOI : https://doi.org/10.7202/200319ar

Aller au sommaire du numéro

\section{Éditeur(s)}

Les Presses de l'Université du Québec

\section{ISSN}

0318-9201 (imprimé)

1705-933X (numérique)

Découvrir la revue

\section{Citer ce document}

Turbide, R. (1982). Michel Tremblay : Du texte à la représentation. Entretien.

Voix et Images, 7(2), 213-224. https://doi.org/10.7202/200319ar d'utilisation que vous pouvez consulter en ligne.

https://apropos.erudit.org/fr/usagers/politique-dutilisation/ 


\title{
Michel Tremblay: \\ Du texte à la représentation
}

\author{
une interview de Roch Turbide
}

Qu'est-ce que le théâtre? Qu'est-ce que la théâtralité ? Ces questions, aussi banales qu'elles puissent paraitre, se posent et doivent encore se poser. Malgré les théories, les discours, les pratiques, on est encore à la recherche de ce qui fait la spécificité du théâtre. Le colloque sur la théatralité tenu en novembre 1980 à l'Université de Toronto a rendu évidentes les divergences entre praticiens et théoriciens, les luttes du professeur et de l'homme de théâtre. C'est avec un esprit curieux, soucieux de se situer au-delà de ces querelles, que nous sommes allé rencontrer Michel Tremblay et l'avons écouté parler de sa vision de la pratique théâtrale.

R.T. Le théâtre est paradoxal parce qu'à la fois production littéraire et représentation concrète. Nous entrerons peut-être au cceur de ce paradoxe en nous posant la question de la spécificité du texte de théâtre. Une pièce de théâtre est-elle pour vous une "histoire" que l'on raconte à un spectateur?

M.T. Certainement pas; d'ailleurs, j'ai beaucoup de difficultés à raconter mes pièces parce que précisément mes pièces n'ont pas d'histoire. Chaque fois que j'ai fait une pièce et que j'ai accordé des entrevues où on me demandait de quoi ça parlait, je pouvais leur répondre, mais je ne pouvais jamais raconter l'histoire. Aucune de mes pièces n'a d'histoire que tu peux raconter. Tu peux dire que Les Bellessœurs est l'histoire d'une femme qui a gagné un million de timbres, que ses voisines viennent lui voler, mais ce n'est pas cela. Dans Hosanna, il y a l'histoire minimale du travesti qui a subi une humiliation, ce qui lui fait découvrir qu'il est idiot d'être quelqu'un d'autre. Je ne peux pas en dire plus car sinon, je vais être obligé de te raconter scène par scène ce qui passe.

R.T. Serait-il plus juste de parler de construction textuelle destinée à un spectateur?

M.T. Oui, dans la mesure où cela fait quinze ans que je dis écrire des fables dans le sens de transposition, soit de raconter une chose en la 
déguisant en autre chose. D'ailleurs, quand je raconte des faits, c'est toujours en récapitulation. Pour moi, une pièce de théâtre, c'est une suite de scènes dans lesquelles il n'y a rien qui se passe, mais dans lesquelles on parle de choses qui se sont passées ou qui vont arriver.

R.T. Mais comment s'assurer que le sens caché que présuppose le terme "fable" soit bien perçu par le spectateur?

M.T. Je ne prends jamais de moyens. J'ai décidé d'écrire pour les gens intelligents, ceux qui veulent comprendre. Je n'impose jamais quoi que ce soit dans mes pièces. Les gens qui ne veulent voir dans Sainte Carmen de la main (parce que c'est la plus "fable" de mes fables) que I'histoire d'une fille qui revient, trouvent ça ennuyant. Il y a des gens qui m'ont dit que les chanteuses-western ne les intéressaient pas. C'est parce que je n'avais pris aucun moyen pour dire et imposer que c'était une pièce sur l'importance de la place de l'artiste dans la société, une pièce sur la culture.

De toute façon, le spectateur n'est pas obligé d'arriver au même message que celui que tu avais l'intention de faire passer. Pour moi, ce n'est pas grave si le spectateur ne va pas jusqu'au $182_{\text {e degré }}$ d'abstraction. Pour Hosanna par exemple, déjà le deuxième degré est intéressant, soit celui du rôle imposé à l'homme et à la femme dans la sociéte; même si tu ne débouches pas sur le thème du québécois déguisé en d'autres choses, tu vas déboucher sur le monde déguisé ou sur les rôles que la société t'impose. Hosanna signifie au Canada anglais tout à fait autre chose. Beaucoup de critiques ont dit qu'Hosanna était le Québec et Cuirette le Canada et que le Québec était fatigué de se faire en... par le Canada. Que veux-tu, c'est leur interprétation; au fond, ce n'est pas si bête, même si ce n'est pas ma vision.

R.T. Vous pouvez tout de même orienter votre spectateur.

M.T. Oui, seulement jusqu'à un certain point. C'est un choix que d'écrire du theâtre. Si je voulais orienter complètement mon spectateur, je ferais moi-même mes mises en scène et ma pièce voudrait dire partout la même chose. La beauté du théâtre, c'est justement le mot "interprète". Quand je vais dans des écoles de théâtre, je leur souligne l'importance de ce mot pour les gens de théatre car ils prennent une matière que quelqu'un d'autre a pensé et ils l'interprètent à leur façon. Par conséquent, c'est très dangereux d'être acteur ou metteur en scène car tu peux détruire tout un texte. C'est comme quand on organise des spectacles pour les jeunes. Je suis absolument contre le fait d'imposer des visions. J'aime mieux un jeune qui comprend croche que celui que l'on fait manger a la petite cuiller. Lors d'une discussion sur Bonjour là, bonjour qu'une école présentait, une vieille dame s'était demandée si les jeunes avaient compris et avait dit qu'il lui semblait que les jeunes auraient eu besoin d'un 
cours avant de voir cette pièce. J'ai alors fait une crise en disant que j'aimais mieux qu'ils ne comprennent pas entièrement, quitte à y revenir dans vingt ans et comprendre autre chose. Cela vaut mieux que de se faire imposer une vision. C'est pour cela que je fais du théâtre. J'ai des affaires à dire, mais je prends des moyens pour m'empêcher d'être trop évident et j'ai un metteur en scène qui coupe les répliques qu'il trouve encore trop évidentes.

Je le répète, c'est un choix d'être un auteur dramatique. Dans un roman, tu imposes ta vision du monde. Si jamais La grosse femme d'à côté est enceinte est traduit, tout le monde va comprendre la même chose parce que j'ai pris un moyen qui est le roman pour m'expliciter. Alors qu'au théatre... Nous jouons Tchekov comme des tragédies bien qu'il ait écrit des comédies. Mais c'est comme cela que nous le comprenons.

Autrement dit, dans un roman, il n'y a personne entre toi et ton lecteur alors qu'au théâtre, tu dépends d'au moins dix ou douze personnes. Tout ce que tu dis est tamisé par les autres; c'est un choix. Mais je trouve cela plus intéressant, plus excitant que d'écrire et de m'expliquer moi-même. Je m'en suis rendu compte quand je suis allé à Vancouver et que des comédiens qui montaient Damnée Manon, Sacrée Sandra m'ont demandé de leur lire le texte en français. J'ai alors lu les monologues de Sandra d'une façon très posée et cela a donné une force à la pièce qu'eux n'avaient pas vue. Quand j'ai vu qu'ils étaient catastrophés, j'ai regretté de l'avoir fait. Ils étaient à Vancouver et c'était leur show. Ils avaient des choses à dire et je n'avais pas à imposer ma vision de la pièce. C'est mon risque.

\section{R.T. Vous faites une grande différence entre roman et théâtre?}

M.T. Oui, en tant qu'outil technique. Quand j'écris du théâtre et quand j'écris un roman, c'est parce que j'ai des choses différentes à dire. J'écris un roman quand je veux parler au monde, moi en tant que moi. Le gars qui fait les descriptions dans la Grosse femme d'à côté est enceinte, c'est moi. C'est moi qui y discute de la religion catholique, ce qu'elle fait aux femmes, alors qu'au théâtre, ce n'est pas nécessairement moi. C'est moi qui écris mais j'emploie le théâtre pour que ça ne soit pas souligné au gros crayon rouge, pour que ça ne soit pas un théorème. En fait, mes pièces ne sont pas des théorèmes. Ce sont les spectateurs qui trouvent le C.Q.F.D. Quand j'écris un texte de théâtre, j'ai toujours conscience que ce n'est pas complet. Je le sais. Pourquoi? C'est peut-être une sorte d'humilité ou peut-être de peur... C'est possible que j'aie peur de dire au monde ce que je pense. D'ailleurs, j'écris moins de théâtre depuis que je suis plus sûr de moi. Tant et aussi longtemps que j'écrirai du théatre, ce sera par humilité et pour me laisser interpréter parce que j'aime me laisser interpréter. J'aime beaucoup. J'ai vu une version de Bonjour là, 
bonjour à Minneapolis où tout se passait dans le décor de Tristan et Isold de Wagner. Pourquoi pas? Moi, je suis très heureux d'avoir des surprises comme ça. Quand j'écris du théâtre, je sais que je me laisse interpréter...

R.T. Et vous faites complètement abstraction de votre propre intention?

M.T. Absolument pas.

R.T. Par quel moyen allez-vous l'imposer?

M.T. Par ma façon d'écrire le théâtre de manière pas réaliste. Dans mes pièces, il y a toujours une dichotomie entre ce que tu vois et ce que tu entends. Ce que tu entends est toujours ou different ou «off» de ce que tu vois, enfin très souvent. Par exemple, tu peux voir physiquement sur la scène des gens assis sur des chaises, mais les actions se passent dans une cuisine que tú ne vois pas nécessairement; c'est une façon de faire passer des affaires que je veux dire aussi. C'est la technique par laquelle je fais passer mes messages. C'est là dans le texte. A cela s'ajoute ce que les autres veulent bien y mettre...

R.T. Si vous le permettez, nous allons revenir au côté technique de l'écriture théâtrale. Même si on disait plus tôt que vos pièces n'ont pas d'histoire, pensez-vous tout de même en termes de "montée dramatique ", de point où tout va changer?

M.T. Bien sûr. J'essaie, dans toutes mes pièces, que le point de départ soit l'endroit où ça commence à changer. Toujours. Dans Bonjour là, bonjour, chaque fois que Serge sonne à une porte et qu'on lui ouvre, il y a quelque chose qui change. La pièce commence comme cela. Dans $\dot{A}$ toi pour toujours ta Marie-Lou, le premier mot de Marie-Lou est “demain». La première chose qu'elle dit est “demain» alors qu'elle et son mari vont mourir le même soir. Le premier mot que j'ai écrit en québécois de ma vie, la première réplique de les Bellessœurs, c'est "Misère moman, qu'est-cé ça?". Les charnières sont toujours dans les premières répliques. Parce que quand je raconte des faits, c'est toujours en récapitulation. Marie-Louise et Léopold qui sont morts depuis dix ans racontent leur vie misérable; les deux filles ne disent rien ou presque.

R.T. Et quel est votre rapport aux personnages? Certains critiques vont vous reprocher parfois de faire des personnages «manqués"; on donne alors souvent l'exemple de Cuirette...

M.T. Mais j'ai fait beaucoup de personnages manqués. Qu'est-ce qu'on a à toujours vouloir que l'on fasse des personnages parfaits? J'en ai fait très tôt. Lisette de Courval est un personnage complètement raté. Elle est très drôle, elle est très ridicule, mais elle n'a aucune espèce d'humanité. Par contre, Cuirette n'est pas un personnage manqué, 
mais un demi-personnage et ce, parce qu'il n'est pas l'objet principal de mon propos. Quand j'ai voulu écrire Hosanna, le titre était "Hosanna et son amant Cuirette» et je l'ai changé pour Hosanna parce que le principal de mon propos était Hosanna et Cuirette n'est là que quand j'en ai besoin. Il est là au premier acte parce que je voulais vider encore une fois l'histoire des rôles imposés par la société à l'homme et à la femme. Tant que j'ai besoin de lui, il est là et même, il apporte parfois des arguments qui sont très pertinents. Hosanna est capable de les détruire parce que c'est un gars, comme il le dit, qui a une grande gueule et qu'il a toujours su s'en servir, mais cela ne veut pas dire que Cuirette a tort. II s'en va quand je n'en ai plus besoin, soit à la fin du premier acte. C'est un personnage-accessoire mais il n'est pas manqué.

R.T. Vous jugez Lisette de Courval par des critères d'humanité. Pourtant, le théâtre n'est pas la vie.

M.T. Il est évident que ça ne m'intéresse pas du tout de faire croire au monde que c'est de la vie. Tu as toujours des moments dans mes pièces où comme déjà dans les Belles-sœurs, et ce même si c'est la première pièce importante que j'ai écrite, la première pièce en québécois, les personnages s'épanchent auprès du spectateur. Par la forme que j'ai donnée à Bonjour là, bonjour, le spectateur sait que c'est un "show" qu'il regarde parce qu'il y a tout au long sept conversations parallèles. Si je m'entends bien avec Brassard, c'est que les spectacles qu'il fait ont la grande qualité d'avoir l'air d'un "show». Tu ne peux jamais oublier que tu es au theatre. D'ailleurs, nous n'avons jamais fait de spectacles «réalistes" sinon notre première version d'En pièces détachées où, même là, on avait réussi à mettre des cages. II y a toujours quelque chose de "théatral» qui fait que le spectateur, même inconsciemment, sait qu'il est au théâtre et ne peut jamais s'imaginer que c'est la vraie vie.

D'ailleurs, tout est techniquement fait de façon à ce qu'aucune interprétation "réaliste» ne soit possible. Par exemple, j'ai vu une version de Hosanna au Café Rimbaud de Québec. C'était une vision très très réaliste d'Hosanna où, au deuxième acte, Hosanna ne parlait pas aux spectateurs. Et je n'ai pas aimé cela. Le comédien était très bon, c'était un excellent spectacle mais je n'ai pas aimé cette interprétation parce qu'ils n'ont pas employé mon moyen technique de dire aux gens qu'ils voyaient du théâtre. Hosanna s'y contait tout à lui-même, ce qui n'était absolument pas lyrique. Je dis lyrique parce que le lyrisme demeure mon grand moyen. Toutes mes pièces sont très près de l'opéra, l'opéra étant pour moi l'absurdité complète, le théâtre parfait. Je trouve que de voir Mimi dans la Boheme mourir consomption dans une mansarde de Paris qui a coûté $\$ 200,000.00$ est le summun du theâtre. On ne peut pas aller plus loin. A l'opéra, tu ne peux jamais oublier que tu es à un "show". Mes pièces, dans cet 
esprit, sont très près de l'opéra. Mis à part l'Impromptu d'Outremont, Hosanna est le meilleur exemple d'une pièce que j'ai écrite où tu ne sais pas si le personnage ne se mettra pas à chanter.

R.T. Et le décor a-t-il une importance pour vous?

M.T. Pas vraiment car je ne suis absolument pas un visuel. Dans les Bellessœurs, c'est écrit «une cuisine». Dans À toi pour toujours ta MarieLou, il n'y a aucune indication de décor. En fait, le décor d'Hosanna est la seule description exacte de décor que j'ai faite. Et c'est parce que moi, j'en avais besoin. C'est que j'avais seulement besoin de visualiser un peu au début; je l'ai oublié après. Cuirette s'accroche dans le «David", puis il parle du tableau mais cela, ce n'est déjà plus le décor. De toute façon, j'aurais très bien pu ne pas le faire publier parce que les décors qui ont été faits de cette pièce n'ont jamais ressemblé à ce que j'avais écrit.

R.T. Est-ce à dire que le monde extérieur, “réel », n'a pas d'importance?

M.T. Pas du tout. C'est que j'essaie plutôt de l'imaginer. C'est ça, un écrivain. Il est là, comme je le dis toujours, pour vivre par procuration, pour vivre à travers les autres. Tu rentres alors dans d'autres mondes et ton talent ou ton habileté te permet à ce moment-là d'interpréter justement des choses que tu ne connais pas exactement. Je décris minutieusement dans Thérèse et Pierrette à l'école des Saints Anges la vie communautaire des religieuses alors que je n'ai jamais rencontré une religieuse de ma vie. Au début, je voulais aller vérifier, me documenter, mais j'étais sûr que je serais beaucoup plus exact en l'inventant. C'est aussi ce que j'ai fait pour Hosanna; je n'avais jamais connu de travestis.

R.T. Dans la mesure où c'est principalement par l'espace que se fait l'articulation texte-représentation, ce rapport à l'espace, tant au niveau du décor que du monde référentiel, devient très déterminant pour le metteur en scène.

M.T. En fait, la grande qualité de mon théâtre réside dans le fait que le metteur en scène peut en faire tout ce qu'il veut. Par exemple, Hosanna n'a jamais été habillée en rouge et or comme je le demandais. On pourrait d'ailleurs se demander pourquoi Hosanna était habillée seulement en or à Toronto tandis qu'elle était, dans la production de Montréal, en or terni.

R.T. Parlant du metteur en scène et de sa liberté, à quel niveau se situe l'apport d'André Brassard? Jusqu'à quel point s'étend votre collaboration?

M.T. Je ne peux pas dire qu'il y a une collaboration entre nous deux. En fait, je sais que si la question semble importante, c'est parce que l'on a longtemps cru que j'étais l'homme de paille de Brassard. Mais il n'en était rien. Tout cela vient du fait que je laissais toujours parler 
Brassard quand venait le temps d'expliquer notre travail parce que les formules qu'il utilisait me paraissaient plus claires que les miennes.

Je dis qu'il n'y a pas de collaboration et j'utilise le pronom «notre" pour parler du travail que nous faisons. II faut bien comprendre qu'il y a un grand nombre d'éléments qui relèvent de ce qu'on pourrait appeler une osmose. La conscience que nous avons du theâtre luimême vient de Brassard alors que la conscience que je pourrais appeler "musicale" vient de moi. Je fais l'intellectualisation de mes pièces, mais c'est lui qui l'explique parce qu'il a plus de facilité à le faire. Par contre, il est vrai que l'on ne peut pas nier une certaine dépendance naturelle que j'avais à l'endroit de Brassard. C'était sans doute dû au fait que je me situe beaucoup plus dans l'emotion que Brassard qui, lui, se situe plus dans les mots. Bref, je crois que l'on pourrait dire que nous avons été pendant longtemps le mentor de l'autre. Jusqu'en 1977, année de Damnée Manon, sacrée Sandra, nous évoluions parallèlement. C'est après que Brassard a pris une autre tangente que la mienne. II ne faut pas oublier non plus qu'une grande partie de cette osmose vient de la nature propre de l'art. L'art est un apprentissage et l'artiste est quelqu'un qui y risque sa vie. Moi, en tant qu'écrivain, je fournis la matière première, mais tous les autres sont aussi créateurs, ont quelque chose à dire. Nous sommes tous, finalement, une bande d'inquiets pris ensemble. Nous avons toujours cherché à travailler en tant que groupe et cela a porté fruit. C'est Hélène Loiselle qui a fait dire à Marie-Lou : « J'ai lu dans le Sélection...».

R.T. Au niveau du travail lui-même, tant la production d'une pièce que son écriture, comment se traduit alors cette osmose?

M.T. Justement, c'est à ce niveau-là que je dis qu'il n'y a pas de collaboration. Bien sûr, du fait que nous soyons liés par l'amitié dépend beaucoup de choses. Par exemple, lorsque j'écris une pièce, Brassard devient un lecteur privilégié ; Il m'aide à jauger l'évidence, la clarté de ma pièce. Mais ses commentaires sont ceux d'un ami, jamais d'un metteur en scène appelé à monter la pièce en question. Il s'en mêle quand il pense que le propos que je tenais n'est plus évident. C'est d'ailleurs facile à constater lorsque Brassard fait une deuxième production d'une de mes pièces. A ce moment-là, Brassard va toujours plus loin dans la facture. La lecture de Brassard y devient beaucoup plus évidente. Par exemple, quand Brassard a repris l'Impromptu au Saidye Bronfman, il a décidé de le monter avec cinq gars. II m'en a parlé, mais je voulais la surprise. II est très excitant de se voir déformaliser. Cela correspond sans doute à un besoin que j'ai de me voir remettre en question.

De toute façon, ces questions que l'on se pose sur la relation Tremblay-Brassard proviennent d'une seule et mauvaise habitude: celle de penser que lorsqu'une pièce est mise en scène, son auteur est mort. Or, jamais pour Brassard, je ne suis mort! 
R.T. Nous avons parlé plus tôt de l'impossibilité au théâtre d'une communication directe. Mais par la forme, la technique, y aurait-il une communication indirecte possible?

M.T. Oui, par le personnage qui arrive sur scène en disant: «je vais vous faire un show dans lequel je vais vous dire ce que l'auteur qui m'a écrit a voulu dire sans le dire exactement". Il y aura aussi les phrases marteaux telle «je suis venue au monde par la porte d'en arrière, je vais donc sortir par la porte d'en avant". Ces phrases, c'est ma façon, mes plus gros trucs, mais il arrive souvent qu'à la représentation, Brassard me les coupe. II pense que cela peut être efficace à la lecture, mais que quand du monde intelligent t'interprète, il y a des choses que tu n'as pas besoin de dire. Les coupures qu'il fait à mes textes sont d'ailleurs toujours à ce niveau-là.

Moi, en tant qu'écrivain de théâtre, je ne veux pas aller plus loin que ce genre de définition qu'est la phrase de Lise Paquette. Exactement comme Manon qui dit qu'elle est une « paillette d'or dans l'œil de Dieu ", réplique qui, selon moi, contient presque toute la signification de la pièce. C'est le plus loin que j'irai pour dire au monde ce que j'ai le goût de dire. Sinon, la pièce deviendrait inutile. J'ai justement arrêté d'écrire principalement pour le théâtre parce que je m'étais assez mis dans Damnée Manon, Sacrée Sandra. C'était pour moi beaucoup. II ne faut jamais oublier que le théâtre est un show. Sinon, je monterais moi-même sur la scène en disant que j'aurais pu écrire une pièce signifiant ceci le monde s'en irait chez eux... et cela coûterait beaucoup moins cher.

R.T. Le silence qui a suivi Damnée Manon, Sacrée Sandra est donc important. Et pourquoi l'Impromptu d'Outremont après ce silence?

M.T. Oui, s'il y a eu un silence après Damnée Manon, c'est que je n'avais plus rien à dire au théâtre. J'avais à dire quelque chose d'autre et c'est pourquoi j'ai écrit des romans. Quant à l'Impromptu d'Outremont, il faut bien comprendre que c'est non pas un accident de parcours comme plusieurs le pensent, mais bien un incident, dans le sens propre du mot, c'est-à-dire tout simplement un fait qui survient au cours d'un fait principal. Trop de gens ont regretté l'ancien cycle. C'est là le problème. C'est peut-être le problème de la critique en soi. La critique doit agir au fur et à mesure que l'œuvre se fait sans savoir vers quoi l'auteur tend, sans avoir, si l'on veut, de perspective. Seul l'auteur est conscient de son cheminement même si dans le cycle des Belles-Sœurs, ce cheminement était évident. Peut-être qu'avec l'Impromptu, je me suis engagé sur une mauvaise voie, voire dans une impasse, mais que l'on attende de voir ce qu'il en sera. II ne faut pas oublier que je travaille toujours à partir d'un propos et ce n'est qu'après que le tout prend forme, que je me demande quel genre de personnages peut illustrer ce que j'ai à dire. 
R.T. II faut donc parler, avec l'Impromptu d'Outremont, d'un nouveau cycle.

M.T. En fait, il faut voir l'impromptu comme un joint, un joint temporaire entre un cycle que j'ai écrit par besoin et un cycle que j'ai eu envie d'écrire. Aujourd'hui, je pourrais dire que je suis un homme de théâtre. Les gens ne se sont pas rendu compte que la seule différence d'avec ce qui précède l'Impromptu est dans le fait que je parle d'un autre monde, d'une couche différente de la société et que finalement, ça revenait tout à fait aux Belles-Sœurs. J'avais tout simplement envie d'écrire sur le retour de la droite et Fernande represente le fascisme que l'on retrouve dans bien des milieux. Et le tout est devenu un impromptu quand je me suis aperçu que je pouvais, moi, au travers ces femmes, dire ce que je ne voulais pas qu'on pense. Je suppose qu'il est normal qu'à mon âge, on ait envie de parler de son métier, de ce qu'on devrait en penser. Car c'est cela: j'ai maintenant envie de parler de ce que je vis. Par exemple, ma prochaine pièce, les Anciennes odeurs, sera précisément sur ce qui me préoccupe actuellement : l'émotion. Auparavant, l'émotion dans mes pièces était individualiste; c'était celle d'un soi face à soi alors que dans cette nouvelle pièce, elle est collective, celle d'un soi face à l'autre. C'est mon propos actuel.

Pour moi, le théâtre exprime le présent alors que le roman regarde le passé. C'est en ce sens que je ne regrette rien de tout ce que j'ai écrit, même plus, que je n'ai rien à renier. En pièces détachées est une pièce qui a très mal vieilli, mais elle a correspondu à quelque chose que je vivais à ce moment-là. Aussi, si j'en reviens à l'Impromptu, j'ai tout simplement eu envie de parler du monde dans lequel je vis présentement. C'est d'ailleurs le seul moyen à ma disposition d'assumer ma récupération. Je suis obligé d'être honnête et c'est en critiquant mon milieu que je peux l'être. Auparavant, j'étais un ouvrier dans un monde ouvrier et j'ai écrit les Belles-Sœurs; je suis obligé d'admettre que je n'y suis plus. Sinon, je ne serais qu'un parvenu. Le plus grand danger pour l'écrivain, c'est l'inconscience.

R.T. Depuis le début de l'entrevue, on parle de trous textuels laissés consciemment dans votre texte, de l'importance du mot "interprète" dans le phénomène théâtral. On souligne l'impossibilité d'une communication directe, la limite d'une communication même indirecte. Quelle serait alors l'utilité d'une pièce de théâtre?

M.T. La pièce de théâtre sert à la discussion qui suivra la représentation. Ça me pue au nez de voir des gens qui sortent du théâtre en disant avoir passé une belle soirée en oubliant ce qu'ils ont vu.

R.T. Quel rôle le spectateur doit-il jouer?

M.T. II doit refaire le puzzle qu'on lui présente. Le theâtre est fait de morceaux que tu donnes au spectateur et que celui-ci doit mettre ensemble. Je hais profondément les publics béats d'admiration, les 
publics paresseux et c'est pour cela que dès les Belles-sceurs, j'ai empêché le spectateur d'être idiot en écrivant des pièces techniquement difficiles. Pas nécessairement difficiles à comprendre mais, par exemple, si tu perds cinq minutes de Bonjour là, bonjour, tu ne comprends plus rien. Le spectateur est obligé d'être intelligent pendant une heure et demie. C'est la même chose pour une pièce comme Hosanna. Si la pièce n'avait pas été techniquement intéressante, je n'aurais pas pu parler pendant deux heures d'homosexualité; les spectateurs seraient partis après les cinq premières minutes. Plus encore, parce que l'on vit dans une société «straight», le spectateur y était obligé, tout au long du premier acte, d'interpréter. Et même les "gais " travaillaient car ils transposaient sans arrêt pour vérifier si ce qui se passait était juste.

R.T. Vous pensez vraiment que le public accepte toujours de travailler?

M.T. Tous les soirs, il y a des idiots qui de toute façon veulent rester idiots. C'était rare qu'il n'y avait pas de rires quand Léopold demandait "Viens-tu faire un tour de machine avec moé à soir Marie-Lou?", comme il y avait toujours quelqu'un qui riait quand André se déshabillait à la fin de Hosanna. Mais si nous vivions dans une societé où tout le monde va au théâtre, on aurait pas eu besoin de ce que j'ai écrit depuis quinze ans. On serait rendu ailleurs, on écrirait autre chose.

R.T. Cela veut dire que vous poursuiviez le but de «populariser " le theatre?

M.T. Bien sûr. J'espère que tout le monde va venir, mais en même temps, je ne peux pas prendre pour acquis qu'il va venir. Le théâtre a toujours été et est encore élitiste. Aujourd'hui, je prends pour acquis qu'ils vont venir en plus grand nombre. Mais ce n'est pas parce qu'ils viennent en plus grand nombre que l'on peut dire que les Québécois vont au théâtre. Ils n'y vont pas encore. C'est en ce sens-là qu'André et moi avons décidé d'être bons, pour tout le monde au lieu d'être géniaux pour nos parents et amis. J'essaie d'écrire pour tout le monde, en sachant très bien que je n'écris pas pour tout le monde. Il faut espérer qu'un joùr viendra...

\section{R.T. Devons-nous parler de frustration?}

M.T. Oui, et c'est terrible. Je peux briser la télévision chaque fois que je vois 60,000 personnes au baseball. Je peux en mourir tellement cela m'humilie. Non pas parce que je condamne le baseball, mais parce que je sais que je ne m'adresserai jamais à 60,000 personnes en même temps.

R.T. Seriez-vous d'accord alors pour dire que la thématique commune des auteurs d'une même époque correspond moins au désir de dire une même chose qu'à l'écoute du public? 
M.T. Si la société n'était pas ce qu'elle est, je n'écrirais pas les mêmes pièces. Je ne pourrais pas dire la même chose. J'écris ce que j'écris parce que je vis dans cette société et que j'en ressens le besoin en même temps que cette société.

R.T. Vous ne vous sentez donc pas en avant de cette société.

M.T. Pas du tout. Je trouve prétentieux et très dangereux de dire qu'un auteur est là pour montrer la voie, surtout pas un auteur de théatre. Un écrivain philosophique, d'accord. L'auteur de théatre est là pour critiquer la société parallèlement à la société. II n'est pas là pour donner des solutions. Je ne suis pas là pour démontrer quelque chose, pour montrer au monde à vivre. Qui suis-je pour montrer le chemin? Je suis là pour me poser des questions avec tout le monde. Puis, nous réglerons nos problèmes tout le monde ensemble.

R.T. Autrement dit, une pièce de théâtre, c'est...

M.T. C'est une question que tu te poses et qui est ensuite reposée par d'autres personnes. Sinon, tu arrives au théâtre de Sartre ou Camus qui sont pour moi du mauvais théâtre. On peut voir bien sûr un certain enseignement lorsque Hosanna dit qu'il faut être soi. Mais c'est aussi un questionnement. Je n'ai jamais écrit quoi que ce soit dans le but d'apprendre des choses au monde. Je pense que je suis là pour canaliser les questions de la société et ce, non pas dans le but que la société change selon mon point de vue. Je n'oblige personne à penser comme moi. Je pense que je dis des choses pertinentes qui demeurent discutables. Tout ce que je dis est discutable.

Ce qui est important, ce n'est pas d'avoir une seule et unique interprétation de la pièce, mais bien que les spectateurs en parlent quand ils quittent la salle. J'ai rencontré des gens qui avaient des raisons absolument géniales de détester ce que je faisais parce qu'ils n'en avaient pas besoin. Tant mieux s'ils n'ont pas besoin de ces questionslà. Si tu n'as pas besoin de mon theâtre, va faire autre chose! Tu seras beaucoup plus utile à la société. Je questionne les spectateurs tout en essayant qu'ils aient du plaisir.

R.T. Justement, on parle de plus en plus de la place du plaisir au theâtre. Vous vous en êtes toujours soucié?

M.T. Dans toutes mes pièces, je veux être "entertaining " dans la mesure où je veux que mon spectateur soit intéressé à la soirée qu'il est en train de passer. Un bon spectacle est celui que tu as du plaisir à voir, qui n'est pas inutile, qui te fait te poser des questions. Exactement comme le bon film est celui que tu n'oublies pas en sortant de la salle.

R.T. Quel est ce plaisir? Comment le créez-vous ?

M.T. Le plaisir est à des niveaux différents. Par exemple, le plaisir de Bonjour là, bonjour est dans la reconstitution constante du puzzle 
ou encore de constater que tu sais, à cause de l'habilité de l'auteur, à qui parle Serge même s'il change presqu'à chaque fois d'interlocuter. Le plaisir d'Hosanna se situe dans l'espèce de concours d'intelligence entre les deux personnages. Le plaisir que crée le premier acte est dans le fait que tu puisses transposer ce qui se dit dans ton propre monde, même si tu ne ressembles pas aux personnages montrés, même s'ils disent des choses très éloignées de toi. Et au deuxième acte, c'est de te laisser emporter par l'opéra, d'en rire ou d'en pleurer. La réplique d'Hosanna quand elle dit que tout le monde était habillé en Cléopâtre est toujours suivie d'un rire monumental. $\mathrm{Ce}$ qui lui arrive étant petit comparativement à ce que ça lui fait, le spectateur trouve la situation tellement ridicule et, comme on dit en anglais, "predictable" qu'il se dit qu'il n'aurait jamais deviné que c'était seulement cela qui s'était passé. Ou encore, le spectateur peut être surpris parce qu'il n'avait jamais pensé au déguisement dans les mêmes termes qu'Hosanna et il se dit alors qu'il est peut-être vrai que c'est aussi terrible qu'elle le dit. Tous les soirs, la salle réagit d'une façon ou de l'autre. Ca aussi, c'est un plaisir. Quant à l'/mpromptu d'Outremont, le plaisir ne sera pas le même pour tout le monde. Certains spectateurs vont faire ce que j'ai fait, soit juger radicalement la société puisque c'est une pièce qui condamne toute bourgeoisie, toute façon élitiste de penser. Par contre, tu as le public qui m'a toujours perçu d'une façon très folklorique en disant qu'il était vrai que je parlais de notre identité et qui aimait ça mais qui ne s'était jamais senti concerné puisque je ne parlais pas d'eux. Avec l'Impromptu d'Outremont, je fais encore des monologues touchants comme j'aime les faire tout en parlant de ce public dont je n'avais jamais parlé et en le condamnant. Ils se sentiront attaqués. De l'autre côté, il y aura les jeunes qui savent que j'essaie de dire des choses et qui auront un plaisir tout à fait différent.

Il existe une autre forme de plaisir que je n'ai pas envie de vendre: les pièces de mots d'auteur. Par exemple, Caligula, les mots d'auteur mis à part, est une pièce que je considère plate. Ton plaisir est d'y entendre de belles phrases sur la vie, plaisir que moi, je trouve insuffisant.

R.T. Ainsi, la lecture idéale de la représentation d'une de vos pièces serait de rechercher la part du plaisir et du message qu'est le questionnement?

M.T. C'est la définition même de tout le théâtre que j'écris. 